\title{
What goes up, must come down
}

The exact functions of sleep are unknown, but it is widely accepted that sleep is important for learning and memory. Two recent studies published in Science found that synaptic connections and synaptic protein markers that respectively increase their numbers and expression levels during wakefulness are downregulated during sleep in Drosophila melanogaster.

Shaw and colleagues investigated the interdependency of increased sleep need after social enrichment and training-induced long-term

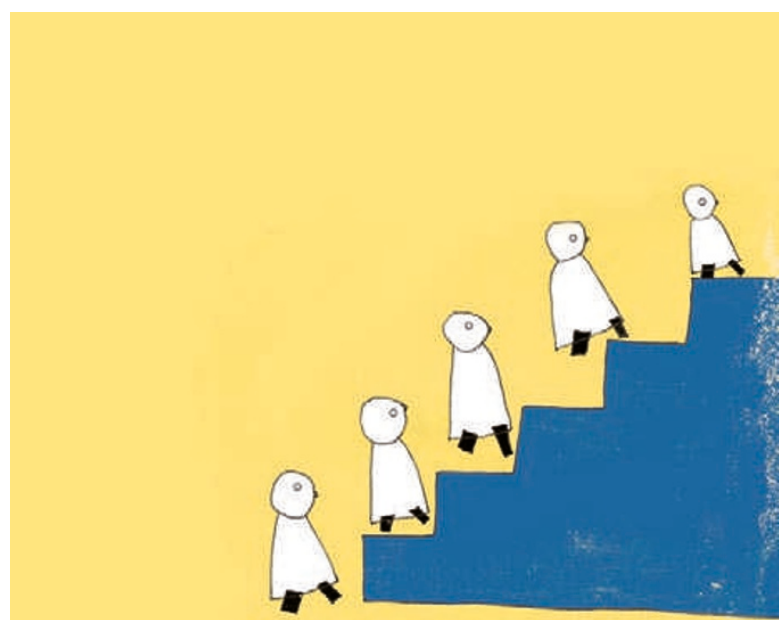

memory. They showed that the number of synaptic terminals on axons projecting from ventral lateral neurons $\left(\mathrm{LN}_{\mathrm{v}} \mathrm{s}\right)-$ clock neurons that regulate the circadian rhythm - to the medulla increased after social experience. The number then decreased during sleep, but it remained unchanged when flies were sleep deprived. Increased sleep need after social interactions or traininginduced long-term memory was not observed in flies that carry mutations in the adenylyl cyclase-encoding gene rutabaga or the clock genes period and blistered (the D. melanogaster homologue for serum response factor). Expression of each of the wildtype genes in the $\mathrm{LN}_{\mathrm{v}} \mathrm{s}$ of the mutant flies rescued this phenotype.

Further evidence of a role for sleep in downscaling synaptic connections that were potentiated during wakefulness is provided by a study by Cirelli and colleagues that investigated the expression of pre- and postsynaptic molecules in the D. melanogaster brain during normal day-night cycles and in flies that were sleep-deprived. The authors found that proteins that regulate the presynaptic secretory machinery or the postsynaptic density increased in expression after periods of prolonged wakefulness. In sleep-deprived flies the levels of these proteins remained elevated, but when flies were allowed to sleep the protein levels declined, suggesting that sleep is required for the downregulation of synaptic proteins. Furthermore, sleep-deprived flies had larger antennal lobes structures that are known to increase in size on activity-induced plasticity — than flies that had slept.

These studies parallel previous studies in the rat suggesting that periods of wakefulness and social enrichment are associated with a net increase in synaptic strength and numbers. Furthermore, they indicate that sleep has a role in downscaling the net increase and thus in normalizing synapses, potentially to keep the system in an 'unsaturated' state.

Claudia Wiedemann

ORIGINAL RESEARCH PAPERS Donlea, J. M. Ramanan, N. \& Shaw, P. J. Use-dependent plasticity in clock neurons regulates sleep need in Drosophila. Science 324, 105-108 (2009)! Gilestro, G., Tononi, G. \& Cirelli, C. Widespread changes in synaptic markers as a function of sleep and wakefulness in Drosophila. Science 324 109-112 (2009) 